\title{
One Step from the Locomotion to the Stepping Pattern
}

\author{
Ronan Boulic \\ EPFL-IIG \\ station 14 \\ 1015 Lausanne \\ 41.21.693.52.46 \\ Ronan.Boulic@epfl.ch
}

\author{
Utku Evci \\ Koç University \\ Rumeli Feneri Mh. \\ 34450 Istanbul
}

uevci@ku.edu.tr

\author{
Eray Molla \\ EPFL-IIG \\ station 14 \\ 1015 Lausanne \\ 41.21.693.52.46 \\ eraymolla@gmail.com
}

\author{
Phanindra Pisupati \\ EPFL-IC \\ station 14 \\ 1015 Lausanne \\ 41.21.693.52.23
}

aditya.phanindra@gmail.com

\begin{abstract}
The locomotion pattern is characterized by a translation displacement mostly occurring along the forward frontal body direction, whereas local repositioning with large re-orientations, i.e. stepping, may induce translations both along the frontal and the lateral body directions (holonomy). We consider here a stepping pattern with initial and final null speeds within a radius of $40 \%$ of the body height and re-orientation up to $180^{\circ}$. We propose a robust step detection method for such a context and identify a consistent intra-subject behavior in terms of the choice of starting foot and the number of steps.
\end{abstract}

\section{CCS Concepts}

- Computing methodologies Motion processing • Computing methodologies Motion path planning • Computing methodologies Motion capture.

\section{Keywords}

Locomotion; Stepping; Holonomic trajectory.

\section{INTRODUCTION}

Locomotion is one of the most used human action in games and simulations involving autonomous virtual humans. However this pattern of movement is characterized by a translation displacement that is tangent to the walking trajectory (non-holonomy) and such a pattern does not necessarily hold for short range displacement involving potentially large re-orientations. Using a standard locomotion pattern in such a context induces noticeable foot sliding that is detrimental for the plausibility of the on-going activity. Proposing a stepping behavior model is a difficult problem as data is scarce and the nature of the body movement is more complex. Indeed the body may move in both the front and the side directions when performing short range re-positioning (holonomy). In this paper we present the first results obtained from the analysis of motion captured data of stepping tasks with null initial and final speeds, for a target positions located within 0.4 body height and reorientations up to $180^{\circ}$ [1].

The paper is organized as follow. First we recall key prior efforts for modeling locomotion. Then we briefly recall the experimental setup before presenting a method for determining the stepping stance phases (period of time when the foot is fixed on the ground). The following section offer our first results in terms of the choice of starting foot and number of steps as a function of the target location. A discussion examine the main direction for future analysis of the data set and conclude the paper.

Permission to make digital or hard copies of all or part of this work for personal or classroom use is granted without fee provided that copies are not made or distributed for profit or commercial advantage and that copies bear this notice and the full citation on the first page. Copyrights for components of this work owned by others than ACM must be honored. Abstracting with credit is permitted. To copy otherwise, or republish, to post on servers or to redistribute to lists, requires prior specific permission and/or a fee. Request permissions from Permissions@acm.org.

CASA 2016, May 23-25, 2016, Geneva, Switzerland.

(C) 2016 ACM. ISBN 978-1-4503-4745-7/16/05 ...\$15.00.

DOI: http://dx.doi.org/10.1145/2915926.2915949
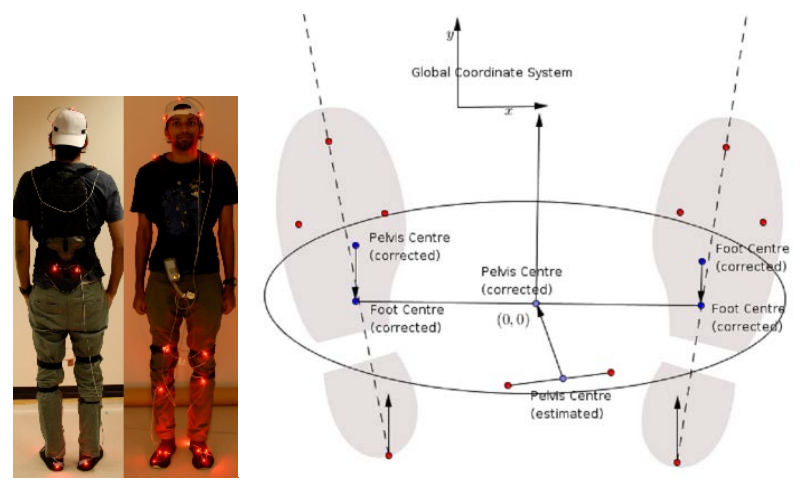

Figure 1. Left: marker setup, Right: top view of the standing-up calibration posture over the origin of the world coordinate system highlighting the four markers per foot and the two markers on the pelvis.

\section{BACKGROUND}

Modeling locomotion for the on-line animation of virtual humans has a long history [2]. Over time the straight-line walking model has been extended to integrate curved walking [3], side and slope walking [4,5]. Footskate is prevented through footprint prediction and fine grain foot trajectory control [5].

A general model of holonomic locomotion control on flat surface has been proposed in Mombaur et al. [6] through the inverse optimization of a cost function weights with an application to humanoid robot control. Despite this achievement more analysis is needed to understand and model the multiple behavioral strategies observed in human subjects when performing the same short range repositioning tasks [1]. For this purpose Pisupati et al proposed an original motion capture setup to ensure an ecologically valid performance of 386 stepping movements with 20 subjects (6 females and 14 males) [1]. The rest of the paper presents how to analyze the captured data to better characterize the stepping behavior.

\section{STEP SEGMENTATION}

\subsection{Feet and Pelvis Registration}

The stepping movements were captured with a Phasespace system and a configuration of 19 markers (Figure 1 left). Each foot was equipped with four markers (three at the front and one on the back of the ankle) allowing to determine a local foot coordinated system without ambiguity (Figure 1 right). The pelvis center and the feet centers were determined from the marker positions in the standing up calibration pose where we assume that the pelvis center projects above the origin of the global coordinate system and that the body front direction is aligned with a predefined orientation. This allows us to compute constant registration transformations that are later 
applied on the captured marker positions to obtain the corrected pelvis and feet centers for the step analysis.

\subsection{Data Pre-Processing}

After the marker position acquisition phase at $480 \mathrm{~Hz}$, the occluded markers were interpolated using cubic interpolation and the resulting data was smoothed using a Butterworth filter of order 3 with a cut-off frequency of $5 \mathrm{~Hz}$ [7].

\subsection{Defining the Stepping Pattern}

Standard walking is characterized by a periodic pattern with clear alternating phases of stance, when the foot is in contact with the ground, and swing, when there is no more contact between the foot and the ground while the leg moves forward to achieve a new step. This distinction is no more possible for stepping as the feet might be sliding while maintaining a contact with the ground to achieve a step. Another frequently observed swing phase behavior is to maintain a point fixed on the ground while rotating the foot around this point. The goal of such swing variants is also to contribute to the body position or orientation reconfiguration while the other foot is totally fixed with respect to the ground in the stance state. Given the rich variety of swing movements we decided to focus on the detection of the stance phases as they are still characterized by a fixed location with respect to the ground to provide a support to the body.

This definition led us to define a criterion for each foot that exploits only the foot movement in the horizontal plane by exploiting its translation speed and its rotation speed. In this paper, we use this criterion in association with a detection automata to filter out most of the micro adjustment steps that some subjects felt compelled to perform, in general but not always at the end of the movement. The output is a first, slightly generous, estimation of the number of stance phases. We present it before discussing why a second level of filtering stage was deemed necessary in the next section.

The criterion $C$ we use for characterizing the stance phase of each foot includes two types of terms:

- A positive term $V$ cumulates the absolute value of the current translation velocity components $\left(v_{x}, v_{y}\right)$ and rotation speed $\omega$ of a given foot.

- A negative term coming from the additive term of the other foot reduces the influence of noise during stance phases and forces an alternating pattern between left and right feet. With such an approach the foot moving the less is forced into a stance state.

Translation is normalized by the body height and evaluated per frame; rotation speed is expressed in radian $(r d)$ per frame. A weighting factor balances the influences of translation and rotation. We have determined empirically the weighting factor of 0.11 to balance the translation and the rotation terms. We have:

$$
\begin{gathered}
V_{R}=\left|v_{x}^{R}\right|+\left|v_{y}^{R}\right|+0.11 *\left|\omega_{R}\right| \\
V_{L}=\left|v_{x}^{L}\right|+\left|v_{y}^{L}\right|+0.11 *\left|\omega_{L}\right| \\
C_{R}=V_{R}-V_{L} / 2 \quad C_{L}=V_{L}-V_{R} / 2
\end{gathered}
$$

\subsection{Stance detection}

The finite-state automaton we use also handles the context of a potentially instable initial state due to the accidental recording of the subject while still adjusting his or her initial location. For this reason we consider that each foot can be in three possible states: unknown (initial state), stance or swing. Transition to the stance or the swing state is made from the comparison of the criterion value to a threshold (Figure 2 and 3) and the confirmation of the new state through a sufficient duration to prevent the creation of micro-states. We retained the durations of 50 frames, equivalent to $\sim 0.1 \mathrm{~s}$, for confirming a stance state (respectively 25 frames for confirming a swing state). The previous state is extended in case a criterion threshold transition is not confirmed by the duration condition such as in Figure 3.

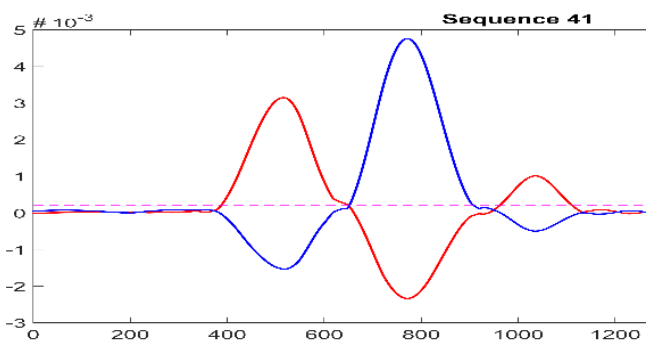

Figure 2. Evolution of the left (blue) and right (red) criteria for classifying the foot state as a swing or a stance state, resp. above or below the dotted line, for a $480 \mathrm{~Hz}$ capture sampling.

An example of identified stances is provided in Figure 4. As one can notice, the end of the sequence still presents some stances that one can consider as adjustments. Further tests on data revealed that increasing the criterion threshold would produce overly long stance states by including also the intermediate swing states where the foot rotates around a fixed point. For this reason we preferred to propose an a posteriori filter at the level of successive stance states of the same foot to remove such adjustments. Basically, a stance is discarded if the normalized distance between two successive stances is lower than 0.07 and the orientation variation is smaller than ten degrees.


Figure 3. Filtering out micro-states due to insufficient duration of the newly detected state.

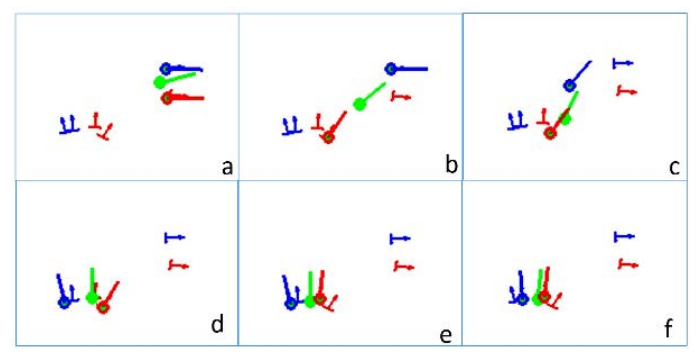

Figure 4. Example of final adjustments that have to be filtered out with the a posteriori stance filtering; red symbols for right stances, blue for left ones; the current state is indicated with the thicker symbols; initial state (a); the subject is moving backward and perform the first right step in (b), the second left step is completed in (d); adjustment steps in (e-f). 


\section{Results}

\subsection{Relation between starting foot and target}

The starting step detected in Pisupati et al. [1] is presented in Figure 8 with the initial location at the center of the plot and the measured starting orientation is aligned with the $\mathrm{Y}$ axis. Each point indicates the final location and is colored either in cyan (right) or magenta (left) according to the starting foot. All subject data are included. Each plot gathers the data having the same theoretical target orientation (presented through two red symbols). Note the variability of final positions that reflect the relaxed performance of the task; this variability is also present in the final orientations. On average, subjects - except one or two - were not biased towards a particular starting foot preference (Figure 5). These plots reveal a dominant pattern of using the starting foot on the same side as the target and independently of the target orientations. Using a Quadratic Discriminant Analysis (QDA) classifier in MATLAB, we were able to characterize the separation contour of the two clusters of points for five levels of probabilities: 0.2, 0.4, 0.5, 0.6 and 0.8 of using the left foot as the starting foot (Figure 8).

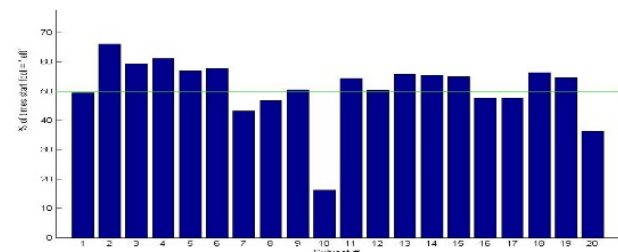

Figure 5. Percentage of using the left foot as starting foot.

\subsection{Number of steps}

The number of steps is given by the total number of stance phases (i.e. from both feet) minus two. We chose to plot the number of steps as a set of Voronoï diagrams where each cell is associated with a single target position and colored according to its number of steps (from dark blue indicating zero step to red indicating five steps). The initial position is located at $(0,0)$ and the initial body orientation is aligned with the $\mathrm{Y}$ axis. Figure 6 displays this information for two subjects and is organized like Figure 8 regarding the target orientation (the Gamma above each plot indicates the orientation variation). For example, the top central plot represents the cases for which the desired final body orientation is the same as the initial one. The plot organization allows to assess whether the behavior is symmetric for opposite gamma values. The limit case of the gamma value of 180 degrees re-orientation leads to a much noisier pattern for some subjects (Figure 6 bottom).

\section{Discussion and conclusion}

The distribution of the number of steps for a given subject shows a rather homogeneous behavior through large areas with the same number of steps both across space and orientation although they performed these movements in a randomized order (intra-subject consistency). On the other hand, Figure 6 also highlights the variability of inter-subject behavior which is significant enough to request a deeper data analysis.

One current direction of our research is to identify stepping strategies explicitly. Indeed, our first observations highlight that multiple strategies are used for the same target as conceptually illustrated in Figure 7. In that figure, the "line of sight" strategy seeks to align the body towards the line linking the body to the target before completing the desired final orientation (dashed line trajectory). On the contrary, in the "shortest path" strategy the body directly starts to rotate towards the final orientation while translating backward (dotted line trajectory). Even more variants are exploited by subjects within the same target region for the same target orientation. We hypothesize that knowing the choice of variant will be sufficient to explain the differing number of steps and to build a model for each of them. Then, on the simulation side, contextual information (nearby obstacles or affordances) will be the key factor for identifying the best variant to achieve a desired repositioning. In some cases, a random choice among multiple possible variants will reproduce the observed inter-subjects variety.

An alternate direction of future work will be to exploit this rich stepping dataset by running some machine learning algorithms at different levels (step number, stance duration and location, feet and body trajectories) to derive a generic model.


Starting position on $(0,0)$

Gamma is the

desired variation

of body orientation

Number of steps armon $1 \mathrm{~m}$
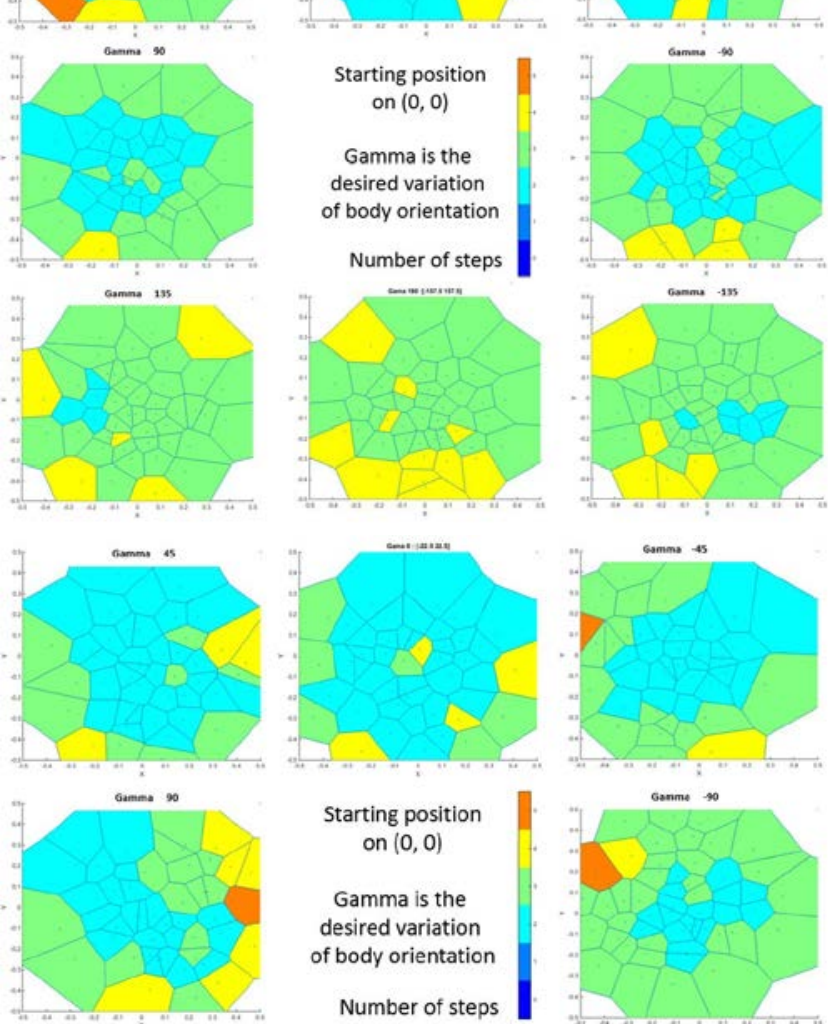

Starting position on $(0,0)$

Gamma is the

desired variation of body orientation

Number of steps
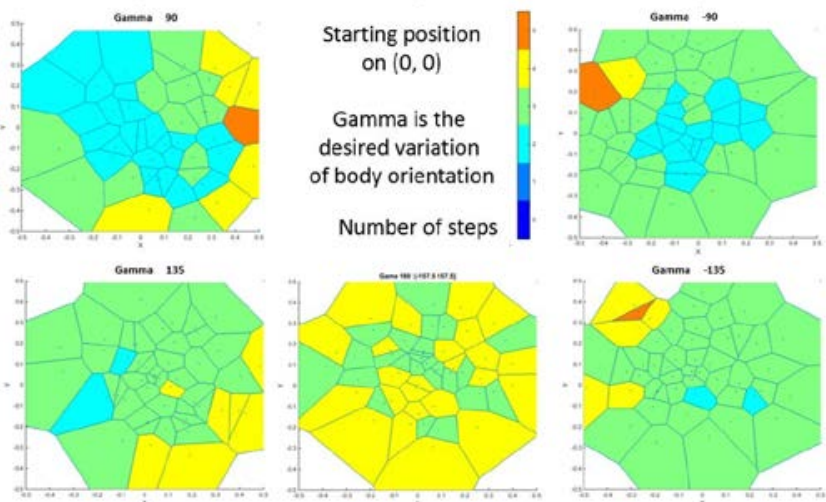

Figure 6. Number of steps for two subjects; each cell in the Voronoï diagrams represent a single target location and is colored according to its number of steps (from zero to five). The target orientation variation gamma is indicated above each diagram. 


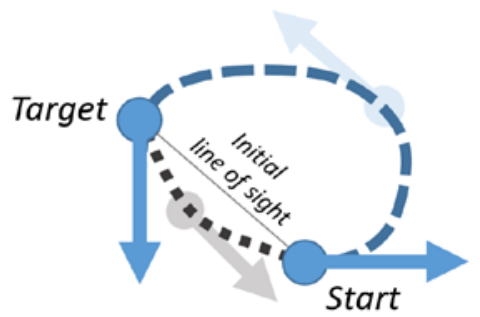

Figure 7. Two frequent stepping strategies.

\section{ACKNOWLEDGMENTS}

This research was partially funded by the SNFS project $\mathrm{N}^{\circ}$ 200020_146827.

\section{REFERENCES}

[1] Pisupati, P., Molla, E., and Boulic, R. 2014. Free-space stepping strategies identification, an exploratory study of short range repositioning. Proceedings of 13th Symposium of 3D Analysis of Human Motion, (Lausanne, Switzerland, July 14-17).3D-AHM2014. 96-99. http://3dahm2014.epfl.ch

[2] Boulic, R., Magnenat-Thalmann, N. and Thalmann, D. 1990. A global human walking model with real-time kinematic personification. Vis. Comput. 6, 6 (Nov. 1990), 344-358. DOI=http://dx.doi.org/10.1007/BF01901021

[3] Pettré, J. and Laumond, J.-P. 2006. A motion capture-based control-space approach for walking mannequins. Comput. Animat. Virtual Worlds 17, 2 (May 2006), 109-126. DOI=http://dx.doi.org/10.1002/cav.v17:2

[4] Feng, A. W., Xu, Y. and Shapiro, A.. 2012. An examplebased motion synthesis technique for locomotion and object manipulation. Proceedings of the ACM SIGGRAPH Symposium on Interactive 3D Graphics and Games (I3D '12), ACM, New York, NY, USA, 95-102. DOI=http://dx.doi.org/10.1145/2159616.2159632

[5] Johansen, R. S. 2009. Automated semi-procedural Animation for Character Locomotion, Master Thesis, Aarhus University

[6] Mombaur, K., Truong, A. and Laumond, J.-P. 2010. From human to humanoid locomotion--an inverse optimal control approach. Auton. Robots 28, 3 (April 2010), 369-383. DOI=http://dx.doi.org/10.1007/s10514-009-9170-7.

[7] Winter, D. Biomechanics and motor control of human movement, ISBN: 978-0-470-39818-0. Wiley.

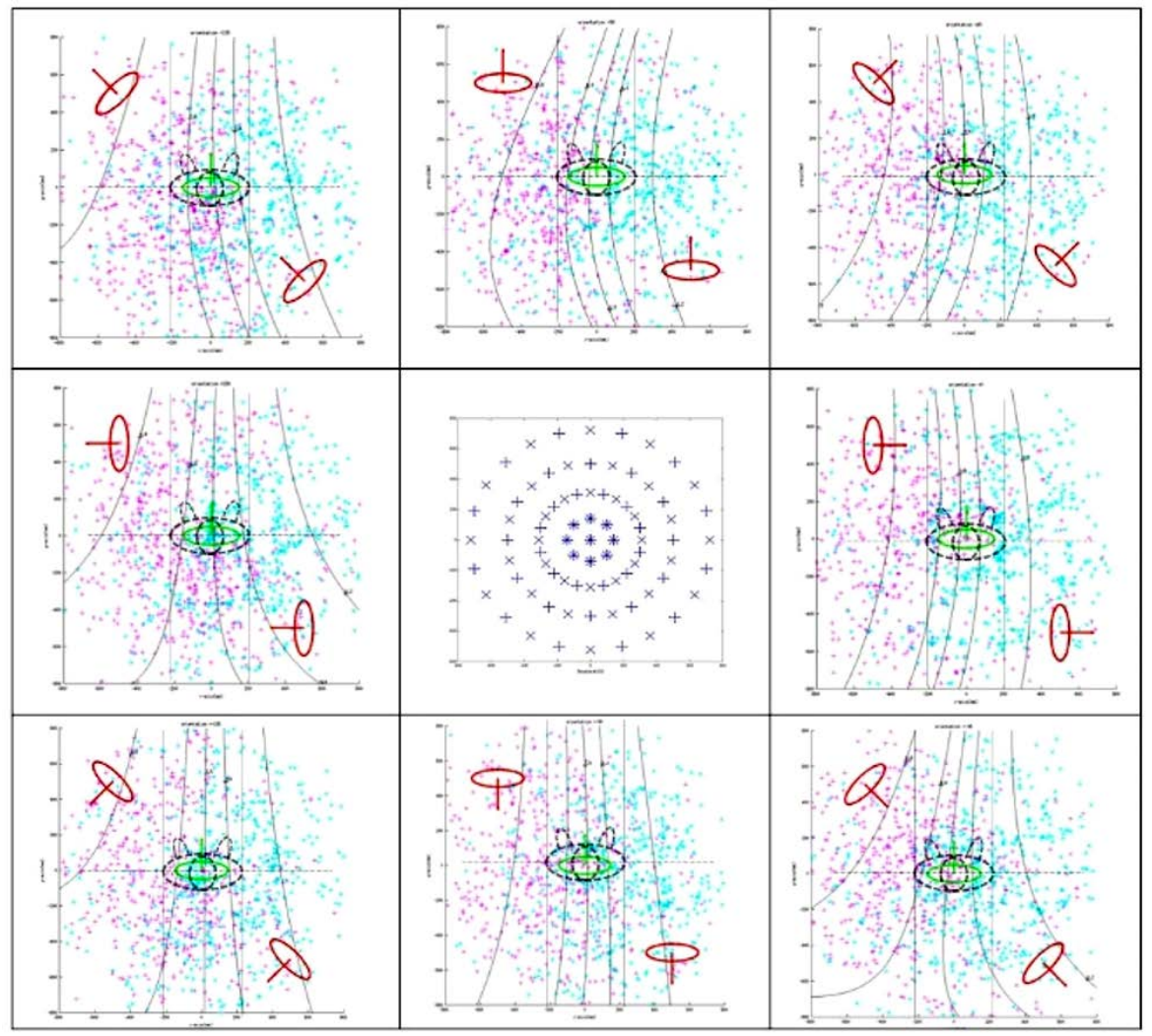

Figure 8. First step detection for the eight possible desired final orientations (indicated by the two red symbols); the body spatial occupancy is indicated by the central grey doted shape. The central image shows the theoretical distribution of desired positions and orientations. A right (resp. left) starting foot is indicated by the cyan (resp. magenta) color of the desired final position. 